\title{
ABORDAJE DE LA CONSCIENCIA CRÍTICA INTERCULTURAL DE GÉNERO DESDE EL MODELO DE UNIVERSIDAD COMUNITARIA E INTERCULTURAL
}

\author{
Sasha Lexei Castillo Ordóñez ${ }^{[1]}$ \\ Sorayda del Carmen Herrera Siles ${ }^{[2]}$ \\ Nuria Gómez Barrio ${ }^{[3]}$
}

\section{Resumen}

La perspectiva de género es uno de los ejes transversales de la Universidad de las Regiones Autónomas de la Costa Caribe Nicaragüense (URACCAN). El estudio tiene por objeto valorar el abordaje de la Consciencia Crítica Intercultural de Género desde el Modelo de Universidad Comunitaria Intercultural, desarrollándose en el marco de una investigación cualitativa, descriptiva y teoría fundamentada.

Los principales resultados señalan que la consciencia crítica de género propone que para lograr transformaciones significativas y profundas de la realidad es necesario tener la capacidad de reconocer las desigualdades que producen la relaciones de poder existentes. Esto implica a la vez varios niveles o categorías, dependiendo estos de la capacidad de apropiación, sensibilización y puesta en desarrollo de acciones transformadoras por la comunidad universitaria.

Palabras clave: Deconstrucción, Educación Intercultural, Interseccionalidad, Pedagogía de la Liberación, Perspectiva Intercultural de Género, SEAR, Teoría Feminista.

\section{Summary}

Gender's perspective is one of the transversal axis in the University of the Autonomous Regions of the Caribbean Coast of Nicaragua (URACCAN). The study aims to assess the approach to gender's intercultural critical awareness from the Intercultural Community University, based on the framework of a qualitative, descriptive and theoretical research.

The main results indicate that gender's critical awareness proposes that in order to achieve significant and profound transformations of the reality it's necessary to be able to recognize the inequalities that are produced through power relations.

[1] Msc. Educación Intercultural Multilingüe. Coordinadora Co-educación CEIMM-Universidad de las Regiones Autónomas de la Costa Caribe Nicaragüense. Bluefields, RAAS. lexeioz@yahoo.com

[2] Msc. Docencia Universitaria. Coordinadora Co-educación CEIMM. Universidad de las Regiones Autónomas de la Costa Caribe Nicaragüense. Siuna, RAAN. barriodolores77@yahoo.com

[3] Msc. Cooperación Internacional Descentralizada. Coordinadora Planificación y Proyectos CEIMM-Universidad de las Regiones Autónomas de la Costa Caribe Nicaragüense. Bluefields, RAAS. nurgobar@gmail.com 
This implies several levels or categories, depending on the capacity of appropriation, awareness and development of transforming actions by the university community.

Keywords: De-construction, intercultural education, inter-sectionality, pedagogy of liberation, gender's intercultural perspective, SEAR, feminist theory.

\section{Introducción}

La URACCAN abre sus puertas en 1995 y plantea, desde el principio ser una universidad inscrita en el marco del reconocimiento y ejercicio de los derechos humanos y colectivos de los pueblos, en el proceso de construcción de la Autonomía Regional Multiétnica de la Costa Caribe Nicaragüense (Marley, 2010). Entre sus ejes transversales incluye género: Forma de promover este enfoque en todo su quehacer institucional. En los últimos años ha incorporado la perspectiva intercultural de género en documentos normativos y ha aprobado una Política Intercultural de Género, a partir de procesos de reflexión, análisis y discusión promovidos en gran medida por su Centro de Estudios e Información de la Mujer Multiétnica (CEIMM).

El Subsistema Educativo Autonómico Regional (SEAR) y la Política Intercultural de Género de la URACCAN, llaman al desarrollo de una Consciencia Crítica Intercultural de Género que conlleve a la transversalización de género, como una forma de superar las brechas de desigualdad entre mujeres y hombres en nuestras comunidades, y que se deben de abordar desde una perspectiva interseccional. "Reconoce que las relaciones desiguales de poder entre hombres y mujeres constituyen un obstáculo crucial para el logro del desarrollo democrático y la lucha contra las causas estructurales de la pobreza y la injusticia social y económica" (URACCAN, 2010a).

Aunque la perspectiva de género no es rechazada explícitamente en el quehacer institucional, se llama a trabajar por su transversalización, pero en la práctica y en el consciente colectivo de la comunidad universitaria hay prejuicios, resistencias arraigadas y persistentes. Esta aseveración la podemos contrarrestar con algunas de las conclusiones arrojadas en el Informe General de la Auditoría de Género de la URACCAN. En él, se concluye que hay cierto nivel de comprensión de la importancia de promover el enfoque de género pero: "discrepancias entre el discurso y lo que en la práctica se está haciendo para promover dicho enfoque". También existe falta de enfoques e instrumentos metodológicos adecuados para darle seguimiento a la aplicación del enfoque de género en los diferentes ámbitos del quehacer académico (URACCAN, 2010b).

El estudio permitió valorar el abordaje de la Consciencia Crítica Intercultural de Género desde el modelo de Universidad Comunitaria Intercultural, lo que implica ir más allá de establecer medidas afirmativas de género o reconocer los derechos de mujeres y hombres. Se debe tener una clara voluntad y acciones que impulsen procesos 
de reflexión sobre la problemática de género, sobre las situaciones de desigualdad y opresión que viven a diario mujeres y hombres, cuestionar las relaciones de poder y proponer acciones para superarlas. Esto supone por tanto una gran ruptura epistemológica, pero también implica transgredir y deconstruir relaciones y el status quo promovidos por la sociedad, que en sí son tareas difíciles de lograr.

\section{Revisión de literatura}

\section{Educación intercultural y enfoque de género}

Las corrientes feministas y agencias internacionales como la FAO (Organización de Naciones Unidas para la Alimentación y la Agricultura; 1998), se refieren a la igualdad de género como: "el compartir por igual el poder de las mujeres y hombres y en esa igualdad acceder a la educación, salud, puestos...." La igualdad de género es una categoría relacional, la "igualdad entre" y no como "igualdad a" (a los hombres). Es un parámetro para tratar a sujetos diferentes como iguales, al tener el mismo valor. La igualdad deseada entre hombres y mujeres es la de sujetos de derechos y no de identidad.

La educación intercultural, promovida desde URACCAN, puede ser entendida como un proceso de carácter social, educativo y político, que tiene como objetivo la construcción de un nuevo paradigma, que supone el replanteamiento de nuevas relaciones sociales basadas en la equidad y en el reconocimiento de identidades y diferencias de los sistemas de conocimientos vigentes (García, Sánchez y Cunningham, 2002).

La interculturalidad debe convertirse en algo más que un referente crítico para examinar las representaciones racistas de la cultura dominante. También debe proponer un espacio en el que la crítica de las prácticas culturales esté ligada a la producción de espacios culturales marcados por la formación de nuevas identidades y prácticas pedagógicas que plantean un vigoroso desafío a los principios clasistas, racistas, patriarcales y sexistas implantadas en la sociedad y la educación (Posada, 2005).

Es así como en su objetivo general, el Sistema Educativo Autonómico Regional (SEAR) contempla: "Promover relaciones de interculturalidad entre hombres y mujeres de las diferentes culturas soportadas en los componentes esenciales de formación de recursos humanos idóneos... que permitan el rescate y promoción de valores positivos de las diferentes culturas de mujeres y hombres..." (IPILC-URACCAN, 2001). Su objetivo seis promueve la equidad de género y dice que el sistema educativo en todos sus niveles tendrá como objetivo: "Desarrollar una consciencia crítica acerca de las relaciones desiguales de género, en particular respecto a la situación que viven las mujeres de los pueblos indígenas y comunidades étnicas" (Ibíd. 6o). Esto se logrará a través de la implementación de medidas positivas que contribuyan a superar las desventajas de las mujeres, currículum con enfoque de género, programas de sensibilización, 
concientización y capacitación a docentes, madres y padres de familia y comunidad en general; asimismo la creación de un instituto de la mujer de la Costa Caribe de Nicaragua.

Sin embargo, entre "los movimientos indígenas y afrocaribeños, la tendencia a una perspectiva sutilmente esencialista hace más fácil la utilización de la "cultura" como pretexto para justificar y reproducir las desigualdades de género". La justificación suele ser que "cualquier cuestionamiento de las tradiciones, de sus prácticas, representaciones, normas, etc., puede ser interpretado como un atentado contra su identidad..." (Girardi, 2004). [...] continúa expresando que: "La interculturalidad es un concepto que se ha trabajado, del que se ha discutido, asociándola con los derechos de los pueblos indígenas y las comunidades étnicas", sin embargo, la autora manifiesta que poco se han:

estudiado las relaciones de género en el contexto multicultural de las Regiones Autónomas y dentro de cada pueblo y comunidad, por lo que se dispone de conocimientos poco profundos y persisten ideas preconcebidas y estereotipos de diferente naturaleza al respecto.

\section{Pedagogía de la liberación}

Ocampo (2008) expresa que la liberación tiene una función política en la sociedad y parte de la realidad de los pueblos de América Latina. En esta concepción de la vida social, la educación se concibe como:

Práctica de la libertad”. La pedagogía freiriana de la liberación, según Roncal (2005) se sostiene en cuatro pilares fundamentales: El carácter fundante del diálogo, la educación como un acto político, aprender es conocer y la perspectiva del conocimiento y los procesos de enseñar y aprender.

Ese diálogo del que habla Freire, para Deere, C. y León, M. (2002) debe ser un diálogo transcultural que también incluya un diálogo interno en las distintas culturas -entre hombres y mujeres-, para producir un consenso en torno a los derechos humanos que emancipe a la humanidad.

La pedagogía de la liberación es un movimiento de acción que rescata la pertenencia a una cultura de los sujetos que reciben educación. Es una pedagogía que critica con acciones pedagógicas y políticas los sistemas educativos, la idea de un sujeto portador de una consciencia unificada, racional y autodeterminante (Arango, 2012).

La universidad, en su posicionamiento filosófico institucional, ha remarcado la importancia de promover esa horizontalidad de la que habla Freire, pero también ese diálogo transcultural entre los pueblos indígenas y comunidades étnicas de la Costa 
Caribe. Sin embargo, también se necesita dar énfasis en esa horizontalidad entre mujeres y hombres de esos pueblos. Es así como se habla de la perspectiva intercultural de género, que implica en sí:

La afirmación de que es necesario un análisis interseccional en la lectura de género, en la que además de incluir otras variables como la ubicación geográfica, clase o edad entre otros, se prime el abordaje desde la cosmovisión, situación y necesidades de las mujeres y hombres de los pueblos indígenas, afrodescendientes y comunidades mestizas de la región (URACCAN-CEIMM, 2010a).

\section{Consciencia crítica y consciencia de género}

Freire formuló una teoría sobre el desarrollo de la consciencia, que contempla tres niveles o categorías. Estos niveles son la consciencia intransitiva, que incluye a las personas que no se preguntan por las causas de los problemas personales y sociales, que no están interesadas en buscarles algún tipo de explicación. La consciencia transitiva ingenua en donde la persona busca las causas de los fenómenos y se interesa por encontrar explicaciones racionales. Por último, la consciencia transitiva o crítica, en el que las personas se esfuerzan por llegar a las causas estructurales de los problemas sociales y a la comprensión de las contradicciones existente, permite la transformación de la realidad (Roncal, 2005).

Mientras que en un estudio de Colas y Jiménez (2006), ellas identifican tres posicionamientos que los sujetos adoptan alrededor de la consciencia de género. $\mathrm{El}$ legitimador implica aceptación y justificación de los modelos de género imperantes, no cuestiona ni ve como problema los roles asignados por sexo. El de resistencia plantea un cuestionamiento de la cultura dominante, cuestiona la realidad de género aunque no plantea propuestas para su cambio. La posición de proyecto implica actitud de construcción de una nueva identidad, busca transformar la estructura social.

\section{Materiales y métodos}

La investigación se realizó en la URACCAN, con presencia física en cuatro recintos en ambas Regiones Autónomas: Bluefields, Nueva Guinea, Bilwi y Siuna; durante los meses de mayo - agosto del 2012. El estudio se enmarca en el paradigma de la investigación cualitativa de tipo descriptivo y se desarrolló a partir del enfoque de la teoría fundamentada.

La población sujeta a estudio fueron las autoridades universitarias, docentes y/o ex-docentes de URACCAN-CEIMM como expertos de trabajo desde la perspectiva intercultural de género, alrededor del abordaje de la Consciencia Crítica Intercultural 
de Género adecuada al modelo intercultural comunitario en el marco del contexto de las Regiones Autónomas.

Se utilizó el método de la teoría fundamentada, la entrevista a profundidad y la revisión bibliográfica como técnicas para la recolección de los datos. Pero, también se aprovecharon momentos claves en el que se abordó el tema de investigación con integrantes del equipo URACCAN-CEIMM (reuniones de equipo). Fue importante para el análisis posterior el haber contado con su percepción sobre el tema.

En el caso de la presente investigación de teoría fundamentada el procesamiento de la información se orientó hacia el desarrollo de una comprensión en profundidad de la situación estudiada basada en los datos brindados por las personas entrevistadas y la revisión de información existente del tema.

\section{Resultados y discusión}

La pedagogía de la liberación, promovida por Paulo Freire, contempla elementos o condiciones para lograr una educación liberadora. Estos elementos o características tienen grandes similitudes en común con lo que define la URACCAN como su modelo de universidad comunitaria intercultural, el cual incorpora la perspectiva intercultural de género.

Hablar de la pedagogía de la liberación desde la perspectiva intercultural de género implica partir del hecho de que en nuestro contexto debemos poder analizar y discutir desde el proceso de régimen autonómico, de la reivindicación de los derechos económicos, sociales y culturales de los pueblos indígenas y comunidades étnicas, pero también desde la lucha por el reconocimiento de los derechos y la autonomía de las mujeres.

Tanto la pedagogía de la liberación como la perspectiva intercultural de género ven la educación como una herramienta de lucha. La educación es política, en palabras de Freire: "el acceso a la educación también es una lucha por la ciudadanía intercultural" (Sichra, 2010), y la transversalidad en la temática de género busca "avanzar hacia la consecución y defensa de los derechos humanos, y hacer frente a las múltiples formas de exclusión que sufren las personas en nuestra sociedad costeña" (URACCAN-CEIMM, 2010a).

\section{Abordaje de la Consciencia Crítica Intercultural de Género en la URACCAN}

Para A. Hooker, el Modelo Pedagógico de URACCAN se basa principalmente en la articulación del conocimiento tradicional y el conocimiento occidental, y para lograr eso en las aulas se fundamenta en el modelo constructivista, quiere decir que debe 
fundamentarse sobre el conocimiento que trae el alumno (comunicación personal, 30 mayo 2012).

Para A. Barbeyto y S. Davis la consciencia crítica de género significa ir más allá y no quedarse sólo con lo que se ve, es trascender y cuestionar con mucha fuerza lo que se ve como normalidad en el mundo sobre los roles de género socialmente establecidos (Comunicación personal, 6 de Junio 2012 y 10 de Junio respectivamente). De manera similar M. Girardi menciona que la Consciencia Crítica Intercultural de Género implica ver el mundo y la vida con otro ojo, leer la realidad de otra manera, comenzar a ver cuáles son las relaciones de poder que existen entre hombres y mujeres... Si no se logra ver esta dimensión de la realidad, se lee de manera distorsionada.

De acuerdo a lo investigado y analizado en el marco de este estudio, dentro y fuera de la universidad se han dado pasos para trabajar desde la perspectiva intercultural de género la consciencia crítica de género. Muchas de estas acciones o procesos han sido impulsados por URACCAN-CEIMM, algunas han tenido mayor impacto que otros, pero todos contribuyen al trabajo a favor de relaciones más horizontales. Pero, también es cierto que todavía persisten, a como es natural, factores que son negativos u obstaculizan el trabajo. A continuación se presentan algunos elementos identificados como dinamizadores y obstaculizadores.

\section{Factores dinamizadores y obstaculizadores sobre la Consciencia Crítica Intercultural de Género}

Se incluye como factor dinamizador los procesos formativos desarrollados por URACCAN-CEIMM. Si bien es cierto que éstos no lograron impactar de igual forma en quienes participaron, se considera positivo que sí se haya logrado el compromiso de algunas personas. Este hecho tiene que ver con las actitudes asimiladas, y no solamente de gestión o manejo de conocimiento.

El trabajo de la URACCAN-CEIMM en particular con las mujeres de y en las comunidades, es una apuesta, un compromiso, que nace desde la fundación misma del centro. Es interesante ver los procesos y los resultados logrados. Nuria Ordóñez menciona el hecho de que las personas que han sido o han tenido la oportunidad de ser educadas a través de procesos promovidos por la universidad, ahora se encuentran ocupando cargos importantes en las Regiones Autónomas. En el caso de las mujeres en las comunidades ahora están en espacios en los que antes sólo había hombres (comunicación personal, 7 de Junio). Para Sandra Davis, el trabajo ha contribuido al desarrollo de la Consciencia Crítica Intercultural de Género entre algunas mujeres indígenas. Son mujeres que aunque no han estudiado están transgrediendo normas muy fuertes, normas culturales y están jugando un rol o asumiendo una consciencia crítica de género. Son valientes por estar planteando cosas diferentes de las que ya están establecidas, y tener la consciencia me parece como un paso positivo (Comunicación personal, 10 de Julio). 
Otro elemento dinamizador de la consciencia crítica de género desarrollado por URACCAN-CEIMM ha sido en palabras de L. Castillo y D. Figueroa:

El buen trabajo con las Comisarías de la Mujer, en comisiones integradas en los municipios (mecanismos para el adelanto de la mujer) y esto ha hecho que se posicione el tema y los debates de la equidad de género y la perspectiva de género.

$\mathrm{Al}$ reflexionar alrededor de este punto, se ve un importante impacto en la sociedad, al visibilizar los temas de género a nivel de las comunidades, municipios y región, en el acompañamiento a estas instancias (comunicación personal 1 y 5 de Julio respectivamente).

Es complejo el hecho de desarrollar una Consciencia Crítica Intercultural de Género en la Costa Caribe de Nicaragua, ya que debes tener en cuenta el componente de la identidad étnica. Esta situación se vuelve más difícil porque las mujeres dentro de estos pueblos tienden a priorizar las demandas de sus colectivos y no valorizan o critican su propia situación como mujeres (S. Marley, comunicación personal, 4 de Junio). Para S. Davis, sí hay transformaciones que se han dado, sin embargo cuesta mucho verlos porque estamos en un mundo de hombres... Las mujeres están trascendiendo ese rol pasivo que hemos tenido las mujeres en las comunidades.

Los limitados recursos humanos con los que cuenta la Universidad para abordar el tema de género de manera especializada es otro factor obstaculizador del proceso. En palabras de M. Girardi (comunicación personal, 7 de Junio 2012), hay dos razones por las que las investigaciones no se hacen con perspectiva de género. Uno de ellos es que la gente no tiene la sensibilidad por lo tanto no les parece importante. La segunda es que la gente no tiene las herramientas necesarias. Estas dos condiciones van juntas. Las afirmaciones anteriores son abordadas en Colas y Jiménez (2006), quienes plantean que: "las transformaciones necesarias para que se desarrolle una auténtica práctica educativa para la equidad requieren del trabajo, de forma consciente y rigurosa, del profesorado. Lo que exige y requiere de «consciencia» y «formación» en género".

Aunque el modelo de la URACCAN llama a insertar, a visibilizar y construir otros conocimientos: "quienes están dando clases full-time ( tigación y por lo tanto difícilmente hacen ese análisis" (S. Marley, comunicación personal, 4 de Junio). Para S. Woods, la Universidad a través de la investigación debe ser capaz de tomar la información de la comunidad y crear teoría a partir de lo que se define como "Grounded theory" [teoría fundamentada] (comunicación personal, 1 de Junio 2012).

Otro elemento que repercute en el desarrollo de la consciencia crítica de género lo representa el hecho que todo el peso de esta temática ha recaído sobre el equipo URACCAN-CEIMM (S. Marley, comunicación personal, 4 de Junio 2012). La afirmación 
anterior es compartida por otras entrevistadas como $S$. Woods quien menciona que en la Universidad al preguntarse acerca del tema suelen decir que es asunto de URACCANCEIMM (comunicación personal, 1 de Junio 2012). D. Figueroa por su parte expresa que: "la gente más fácil digiere el discurso de la interculturalidad que el de la justicia de género, y es más fácil que la gente se comprometa a hacer activismo intercultural que activismo por la equidad de género y feminista." (Comunicación personal, 5 de Julio 2012).

\section{Consciencia Crítica Intercultural de Género y sus niveles-categorías}

Por medio de un análisis comparativo de las teorías freiriana, de género e interculturalidad, y del análisis de la información obtenida de las entrevistas realizadas en el marco del estudio, se propone desde el Modelo Pedagógico de la URACCAN desde la perspectiva de Consciencia Crítica Intercultural de Género y sus niveles-categorías.

La Consciencia Crítica Intercultural de Género es entonces la capacidad de lectura analítica que permite reinterpretar la realidad deconstruyendo desde una perspectiva interseccional los discursos homogenizantes, que las culturas dominantes construyen entre los pueblos y a lo interno de los mismos, para invisibilizar a las mujeres y naturalizar las diferentes formas de desigualdad en función de legitimizar las relaciones de poder. Esta deconstrucción implica a la vez la construcción de nuevas identidades y relaciones de género e interétnicas desde la reconceptualización holística de la realidad y cosmovisión de cada uno de los pueblos como elemento básico para la construcción de ciudadanías interculturales de género.

La Categoría Invisibilizadora es el análisis parcial de las realidades que no permite la identificación de las diferentes formas de desigualdad entre ellas: las de género y étnicas, negando por tanto las medidas de acción afirmativa y legitimizando el status quo establecido. Categoría Discursiva es el análisis consciente de la existencia de conflictos de poder en las relaciones de género y étnicas con un compromiso basado fundamentalmente en el discurso y no en el accionar. En el caso de proponer ciertas medidas de intervención en base a acciones afirmativas no necesariamente integrales ni transformadoras, y generalmente se centralizan en una dimensión (étnica o de género sin llegar a un abordaje articulado de ambos desde un análisis interseccional). La categoría Deconstructiva-Constructiva es el análisis crítico de las realidades de las mujeres y hombres de los diferentes pueblos desde un enfoque interseccional que reconoce las diferentes formas de desigualdad, deconstruyendo los modelos dominantes y homogenizantes, y proponiendo e implementando acciones transformadoras pertinentes que contribuyen al ejercicio efectivo de los derechos colectivos e individuales. 


\section{Acciones para desarrollar la perspectiva intercultural de género en el SEAR}

Un aporte importante se dio desde el proceso de elaboración del Sistema Educativo Autonómico Regional (SEAR) entre el 2000- 2001. URACCAN-CEIMM jugó un papel importantísimo porque se incluyó la perspectiva de género en el SEAR, que nace desde el SEAR que es el sistema educativo de la región, por tanto, cualquier proceso que la Universidad haya dirigido a su interior o hacia afuera para desarrollar la perspectiva intercultural de género, contribuye a operativizar el SEAR. Si solamente la URACCAN hiciera un trabajo acucioso, planificado hacia adentro, estaría dando un aporte espectacular a la operativización desde la perspectiva de género en SEAR (Girardi, comunicación personal, 7 de Junio 2012).

Otros aportes extraordinarios de la Universidad han sido en la investigación no sólo educativas, sino también en temáticas específicas, es como un eje fundamental del aporte de la perspectiva de género del SEAR. La otra son capacitaciones con la comisión de educación, con los docentes de primaria, secundaria, el acompañamiento en la elaboración y readecuación currícular.

\section{Propuesta teórica-metodológica de la Consciencia Crítica Intercultural de Género}

La perspectiva intercultural de género y la pedagogía de la liberación comparten elementos en común,mas allá de las que puedan diferir. Hablamos de cuestionar las relaciones desiguales que se dan en nuestras sociedades y sus estructuras; pero, también la búsqueda del respeto, la visibilización y revalorización del conocimiento propio de mujeres y hombres, la creación de espacios de diálogo y relaciones de horizontalidad. Esta consciencia crítica del que habla Freire, también se busca desde la perspectiva intercultural de género, ya que es una lucha que se inserta en el proceso autonómico regional. La Universidad como institución autonómica debe de ver la educación como el acto político de formar a costeñas y costeños con una lectura crítica y propositiva de los fenómenos sociales dentro de la región y del país.

Es necesario que todas las áreas académicas, centro e institutos puedan dar propuestas concretas sobre cómo abordar la perspectiva intercultural de género en sus espacios, organizar sus temas de acuerdo a sus demandas y necesidades. Esta reflexión debe conllevar procesos de toma de consciencia, de generación de otros conocimientos. Así lo expresa S. Davis al decir que la gente no está entiendo la necesidad de hablar este tipo de temas, y sí hay que cambiar un poco el pensamiento y resistencia de la gente, no hay mucho compromiso (comunicación personal, 10 de Julio). Para S. Woods esto tiene que ver también con un problema de recursos económicos, ya que para ella trabajar las investigaciones y demás procesos desde la perspectiva intercultural de género requiere más recursos económicos y tiempo (comunicación personal, 1 de Junio 2012). El problema para A. Barbeyto es: "transcender del discurso a la práctica, 
y también creo que hay gente que no está entendiendo el pensamiento crítico de género, que están en espacios de toma de decisiones, creo que ese es el gran problema".

Para S. Davis, la falta del paso del discurso a la práctica tiene que ver con el hecho de que hay mucha gente que tiene resistencia a las acciones afirmativas que se necesitan promover en la Universidad. Ella hace un llamado a la comunidad universitaria y sobre todo a los que dirigen en el deber a informarse más acerca del porqué de la "acción afirmativa", es decir, sensibilizarse sobre el tema (comunicación personal, 10 de Julio 2012).

La Universidad está cada vez convencida que debe articular saberes con las comunidades locales y habría que ver cómo empoderar los saberes de las mujeres y no solamente los saberes colectivos, y esta información debe regresar a la comunidad. Este proceso debería de ir cambiando las actitudes para con las mujeres para entender mejor el porqué de sus demandas (S. Marley, comunicación, 4 de Junio 2012). Para M. Girardi (comunicación personal, 7 de Junio 2012), visibilizar los conocimientos no oficiales le da fuerza a los sujetos que son portadores de esos conocimientos.

\section{Abordaje de la Intercultural de Género desde la visión interseccional}

La Universidad debe tener acciones afirmativas que permitan tener más personal con capacidad de reflexionar críticamente sobre interculturalidad y género y asegurar que transmiten estos saberes en el aula de clase [...] se aplican en sus investigaciones y que proponen conceptos o elementos teóricos para profundizar en estas cuestiones. (S. Marley, comunicación personal. 4 de Junio 2012). Para N. Ordóñez, la Universidad ha producido muchas investigaciones, muchos conocimientos de cada uno de los pueblos, pero hace falta dedicar más esfuerzo en asegurar que esas investigaciones se conviertan en contenidos programáticos para el currículo y asegurar que las investigaciones sean parte de las bibliografías que los estudiantes utilicen (comunicación personal, 7 de Junio 2012).

S. Marley aboga porque sean los docentes quienes identifiquen la necesidad de avanzar en discusiones más teóricas que no tengan que ver sólo con lo aplicado propiamente al área de ellos, sino en entender por qué género, por qué hablamos de las discriminaciones y que vean el género como parte de la sociedad (comunicación personal, 4 de Junio 2012). Esto se refuerza con lo expuesto por Colas y Jiménez (2006), quienes propugnan que "a la docencia le corresponde la tarea de pensar su enseñanza con una nueva visión de género y crear un nuevo marco de actuación educativa adaptado a su propio contexto y alumnos/as". 


\section{Propuesta teórica metodológica para consolidar la interculturalidad de género}

Consolidar la Consciencia Crítica Intercultural de Género en la URACCAN, enfocada a la identificación, crítica y deconstrucción de las desigualdades de género e interétnicas, así como también las relaciones de poder desiguales, con el fin de poder construir nuevas alternativas educativas orientadas a la erradicación de todas las formas de opresión y desigualdades. Se plantea, para ello, el desarrollo de las siguientes estrategias:

- Formación diferenciada de docentes e investigadores en dos niveles: sensibilización, concientización.

- Formación diferenciadas de docentes e investigadores en capacitación- especialización.

- Diseño de herramientas metodológicas.

- Optimización de herramientas.

\section{Conclusiones}

El grado de apropiación de la Consciencia Crítica Intercultural de Género en la URACCAN, se manifiesta en diferentes niveles, por lo que es necesario desarrollarla de manera más amplia y profunda. El equipo de URACCAN-CEIMM, por su naturaleza tiene un mayor grado de acercamiento, especialización del tema y aplicación a través de su accionar en docencia, investigación y extensión comunitaria. Algunos de la comunidad educativa se encuentra entre las categorías invisibilizadoras y discursivas.

En la URACCAN es más fácil abordar crítica y constructivamente la perspectiva intercultural que hacerlo desde la perspectiva de género, este rechazo tiene raíces en el hecho imaginario de equivaler género y mujer. Este discurso es promovido por el sistema patriarcal dentro de los pueblos indígenas y comunidades étnicas, aludiendo a que los temas de género, feminismo, entre otros, son ajenos a su cultura y debilitan o destruyen la identidad.

La resistencia hacia el accionar transformador que propone la Consciencia Crítica Intercultural de Género contradice el discurso oficial de la Universidad (plasmado en una Política Intercultural de Género).

Para lograr transformaciones profundas, las autoridades y docentes deben adoptar posiciones comprometidas a cambiar actitudes y prácticas que legitiman las relaciones desiguales basadas en el sistema sexo-género que legitiman el status quo. 
Los aportes de la URACCAN al cumplimiento del SEAR por medio del desarrollo de la perspectiva intercultural de género suelen ser identificados desde las acciones que realiza hacia fuera. Sin embargo, la mayoría de su quehacer está en concordancia desde la formación de recursos humanos idóneos, el fortalecimiento de las identidades de los pueblos, la creación de fuentes materiales e inmateriales de conocimiento, todos estos elementos contribuyen a ser realidad el proyecto autonómico.

\section{Lista de referencias}

Arango, S.(2012). Pedagogía feminista: Una lectura desde la crítica cultural feminista. IX Simposio Internacional Educación y Cultura en Iberoamérica. Universidad de Ciencias Pedagógicas “Juan Marinello Vidaurreta”, Matanzas, Cuba, 20-24 Marzo. Consultado el 4 de Junio, 2012 en http://casimentiras.wordpress.com/2012/05/o7/ pedagogia-feminista-una-lectura-desde-la-critica-cultural-feminista/

Colás, P., Jiménez, R. (2006). Tipos de consciencia de género del profesorado en los contextos escolares. Revista de Educación, 340. 415-444pp. Recuperado el 11 de Junio, 2011 en www.revistaeducacion.mec.es/re340/re340_15.pdf

Deere, C y León, M. (2002). En defensa de la comunidad: Luchas étnicas y de género en torno a los derechos individuales y colectivos a la tierra. 163-217p en: Deere, C. Género (2002), Propiedad y empoderamiento: Tierra, estado y mercado en América Latina. México D.F.: Universidad Nacional Autónoma de México: FLACSO Sede Ecuador. 502 p.

Organización de las Naciones Unidas para la Alimentación y la Agricultura (FAO, 1998). Censos Agropecuarios y Género. Conceptos y Metodología. Recuperado el 10 de Junio de 2011 desde www.fao.org/docrep/o04/x2919s/x2919soo.htm

García, A., Sánchez, T. y Cunningham, M. (2002). Estrategias para la incorporación de la interculturalidad como eje transversal en URACCAN.

Girardi, M. (2004). Relaciones de género en la educación intercultural bilingüe.Diagnóstico de las relaciones de género en la escuela Winston Hebbert, Bilwi, RAAN. Validación de una metodología. Tesis de maestría no publicada, Universidad Centro Americana, Managua, Nicaragua..

Instituto de Promoción e Investigación Lingüística y Rescate Cultural (IPILC-URACCAN). (2001). Sistema Educativo Autonómico Regional. Consejo Regional Autónomo RAAN-RAAS. 
Marley, S. (2010). La Perspectiva Intercultural de género en el Marco de un Modelo de Universidad Comunitaria: Lecciones Aprendidas y Desafíos. Seminario Perspectiva Intercultural de Género en la Educación Superior. Ed.Sichra,I. 81-9o. Cochabamba Bolivia, 2009.

Ocampo, J. (2008). Paulo Freire y la pedagogía del oprimido. Revista Historia de la Educación Latinoamericana, Núm. 010. Universidad Pedagógico y Tecnológica de Colombia. Tunja, Colombia. Pp 57-72.

Posada, J. (2005). El pensamiento de Paulo Freire y la construcción de propuestas pedagógicas y curriculares para la Interculturalidad. En: Robles, O. (2007) Tras las Huellas de Paulo Freire. Compilación. Managua, Nicaragua. 123-145 p.

Roncal, F. (2005). Recopilador. Educación para el sentido crítico. Diplomado en innovaciones educativas y aprendizaje. Curso 7. Guatemala. 130 pp.

Sichra, I. (2010). Seminario Perspectiva Intercultural de Género en Educación Superior. Intercambio de Experiencias y Construcción de Propuesta. Nicaragua, Bolivia, Noruega. Cochabamba, Bolivia. 19-21 de Octubre, 2009. 228 pp.

URACCAN-CEIMM. (2010a). Política Intercultural de Género de URACCAN.

URACCAN-CEIMM. (2010b). Informe de Auditoría de Género URACCAN. 